\title{
Cinobufacini-induced HeLa cell apoptosis enhanced by curcumin
}

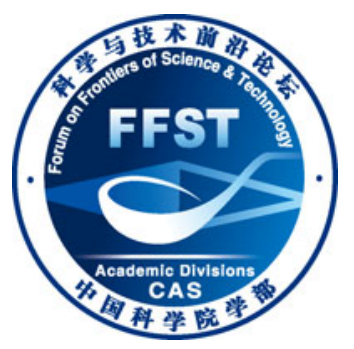

\author{
LIU Li $^{1 \dagger}{ }^{\dagger}$ JIN Hua $^{1 \dagger}$, OU JinLai ${ }^{2}$, JIANG JinHuan $^{1}$, PI Jiang ${ }^{1}$, KE ChangHong ${ }^{1}$, YANG Fen ${ }^{1}$, \\ QIAO DongJuan ${ }^{1}$, CAI HuaiHong ${ }^{1} \&$ CAI JiYe ${ }^{1 *}$
}

${ }^{1}$ Department of Chemistry and Institute for Nano-Chemistry, Jinan University, Guangzhou 510632, China;
${ }^{2}$ Department of Pharmaceutics, College of Pharmacy, Jinan University, Guangzhou 510632, China

Received October 28, 2012; accepted January 5, 2013; published online April 10, 2013

\begin{abstract}
When used in combination with certain chemotherapies, curcumin has been shown to increase apoptosis in several cancer cell lines. Here, we report the combined effects of curcumin and cinobufacini on human cervical carcinoma cells. The aim of this study was to examine whether curcumin could enhance apoptosis induced by cinobufacini. 3-(4,5-Dimethylthiazol-2-y1)-2,5diphenytetrazolium bromide (MTT) assays revealed that the growth and proliferation of HeLa cells could be inhibited by $75 \%$ after a combined treatment of $25 \mu \mathrm{g} / \mathrm{mL}$ cinobufacini and $8 \mu \mathrm{g} / \mathrm{mL}$ curcumin. The combined treatment is 3 times more effective than treatment with $25 \mu \mathrm{g} / \mathrm{mL}$ cinobufacini alone. Annexin V-FITC/PI staining, morphological changes and immunofluorescence verified a significant enhancement in cinobufacini-induced apoptosis when cells were also exposed to curcumin. The data showed that the proportion of early apoptotic cells significantly increased from $15.43 \%$ in cells treated only with $25 \mu \mathrm{g} / \mathrm{mL}$ cinobufacini to $49.2 \%$ in cells treated with $25 \mu \mathrm{g} / \mathrm{mL}$ cinobufacini and $8 \mu \mathrm{g} / \mathrm{mL}$ curcumin. Moreover, compared with treatment of only $25 \mu \mathrm{g} / \mathrm{mL}$ cinobufacini, ROS production increased 1.7-fold, the intracellular free $\mathrm{Ca}^{2+}$ concentration increased 1.5-fold, and the mitochondrial membrane potential decreased by $20 \%$ in the combined treatment. Simultaneously, the atomic force microscopy (AFM) results suggest that cells treated with a combination of cinobufacini and curcumin varied significantly in shape and ultrastructure. Collapsed cells with leaking cytoplasm, blebbing pores and emerging apoptotic bodies were prevalent. The nanoparticle size increased from $70 \mathrm{~nm}$ when the cells were treated with $25 \mu \mathrm{g} / \mathrm{mL}$ cinobufacini to $190 \mathrm{~nm}$ when the cells were treated with $25 \mu \mathrm{g} / \mathrm{mL}$ cinobufacini and $8 \mu \mathrm{g} / \mathrm{mL}$ curcumin. The size increase resulted in the cell membrane becoming considerably rough. These results can improve our understanding of combination treatments. Specifically, the combination of cinobufacini and curcumin may potentially find use as a novel cervical carcinoma treatment. Additionally, AFM is a powerful tool that can be used to explore cellular morphologies and ultrastructures.
\end{abstract}

apoptosis, HeLa cells, AFM, ultrastructure, ROS, MMP

Citation: Liu L, Jin H, Ou J L, et al. Cinobufacini-induced HeLa cell apoptosis enhanced by curcumin. Chin Sci Bull, 2013, 58: 2584-2593, doi: 10.1007/s11434013-5739-9

Cervical carcinoma is the third most common cause of female mortality. A number of chemotherapeutic agents, including cisplatin [1,2], paclitaxel [3], ifosfamide [4], and topotecan [5], have shown activity against advanced and metastatic cervical cancer. Compared with single-agent cisplatin, cisplatin-based combination chemotherapy improves patient survival; however, this combination therapy shows

$\dagger$ These authors contributed equally to this work.

*Corresponding author (email: tjycai@jnu.edu.cn) greater toxicity and the improvements are only modest [6]. Therefore, there is a clear need for the development of new agents with novel mechanisms of action against this disease. In this study, we combined cinobufacini (CIN) and curcumin (CUR) to treat cervical carcinoma.

Recently, several traditional Chinese medicines with anti-tumor properties have attracted considerable interest as candidates for novel cancer therapeutics [7]. CIN is a watersoluble extract prepared from the skins of Bufo bufo gargarizans. Recent clinical studies have suggested that CIN, 
used alone or in combination with other chemotherapeutic agents, has a profound effect on a number of cancers, particularly gastrointestinal tract carcinomas such as liver, pancreatic [8], and gastric cancers [9,10].

CUR is a low molecular weight and natural polyphenolic compound isolated from the turmeric rhizome (Curcuma longa). CUR has been reported to harbor anti-oxidative, anti-inflammatory, anti-angiogenic, anti-proliferative, antitumor and wound healing properties [11-15]. CUR induces cancer cell apoptosis and shows low cytotoxicity in normal cells $[16,17]$.

However, there are no reports on the combined effects of CIN and CUR on cervical cancer cells. The aim of this study was to examine whether CUR enhances CIN-induced apoptosis. We first used HeLa cervical cancer cell lines as a model to investigate CUR enhanced CIN-induced apoptosis. Simultaneously, an 3-(4,5-dimethylthiazol-2-y1)-2,5-diphenytetrazolium bromide (MTT) assay, immunofluorescence, and flow cytometry were used to analyze the underlying molecular mechanisms of this enhancement. In recent years, attempts to understand the cellular, subcellular and molecular mechanical changes that occur throughout human disease states, including cancer, have garnered significant scientific interest. The plasma membrane, which is the boundary between a living cell and its environment, plays a very important role in cellular physiology. The plasma membrane protects the cell [18] and regulates cellular functions, including the transport of nutrients $[19,20]$. Understanding the relationships between cellular ultrastructures, cellular and cytoskeletal mechanical properties, biological function and human health and disease states is a research area that has garnered much attention [21]. In this paper, we used AFM to visualize nanoscale changes in the subcellular ultrastructure resulting from chemical treatment.

\section{Materials and methods}

\subsection{Reagents and cell culture}

CIN was obtained from Anhui Jinchan Biochemical Co. Ltd. (Huaibei, China). CUR was purchased from Tianjin Yongda Chemical Reagent Development Center (Tianjin, China). CUR was initially dissolved in dimethyl sulfoxide (DMSO) to obtain the desired concentrations. Fetal bovine serum, RPMI-1640, trypsin, and MTT kit was obtained from Gibco (New York, USA). An Annexin V-FITC/PI apoptosis detection kit, a Rhodamin 123 kit, a Fluo-3 AM kit, a 2',7'-dichlorofluorescein diacetate kit, a 5,5',6,6'-tetrachloro-1, $1^{\prime}$, 3,3'-tetraethyl-imidacarbocyanine iodide (JC-1) kit, a 2-(4amidinophenyl)-6-indo-119 lecarb-amidine dihydrochloride (DAPI) kit, and fluorescein isothiocyanate (FITC)-phalloidin were purchased from Beyotime, China. All reagents used in the experiments were analytical grade. The ultrapure water used for all experiments was supplied by the cascade RO water purification system from Pall (New York, USA).
The HeLa cells were purchased from the Life Science Research Institute of the Cell Resource Center in Shanghai, China. The cells were cultured in RPMI-1640 media supplemented with $2 \mathrm{mmol} / \mathrm{L}$ glutamine, $10 \%$ fetal bovine serum, $100 \mathrm{U} / \mathrm{mL}$ penicillin and $100 \mathrm{~g} / \mathrm{mL}$ of streptomycin, and the cultures were grown at $37^{\circ} \mathrm{C}$ in a humidified atmosphere containing $5 \% \quad \mathrm{CO}_{2}$. When the cells grew to $70 \%-80 \%$ confluence, they were passed according to a $1: 3$ split. Once in the logarithmic growth phase, the cells were ready for the following experiments.

\subsection{MTT assay}

An MTT assay was employed to assess the toxicity effects of CIN alone or in combination with CUR on HeLa cells. Briefly, cells $\left(6 \times 10^{4}\right.$ cells $\left./ \mathrm{mL}\right)$ were plated in 96 -well plates. After $24 \mathrm{~h}$, the cells were incubated with different concentrations of CIN $(0,6.25,12.5,25,37.5$ and $50 \mu \mathrm{g} / \mathrm{mL})$ and either $4 \mu \mathrm{g} / \mathrm{mL}$ of CUR $\left(\mathrm{CUR}_{4}\right)$ or $8 \mu \mathrm{g} / \mathrm{mL}$ of CUR $\left(\mathrm{CUR}_{8}\right)$. The combined assay employed either $25 \mu \mathrm{g} / \mathrm{mL}$ CIN and $4 \mu \mathrm{g} / \mathrm{mL}$ CUR $\left(\mathrm{CIN}_{25} / \mathrm{CUR}_{4}\right)$ or $25 \mu \mathrm{g} / \mathrm{mL} \mathrm{CIN}$ and 8 $\mu \mathrm{g} / \mathrm{mL}$ CUR $\left(\mathrm{CIN}_{25} / \mathrm{CUR}_{8}\right)$; the cells were incubated with the agents for $48 \mathrm{~h}$. Then, MTT was added to a final concentration of $10 \mu \mathrm{g} / \mathrm{mL}$ in each well for a 4-h incubation at $37^{\circ} \mathrm{C}$. The medium was then removed, and the cells were suspended in $150 \mu \mathrm{L}$ of DMSO for $15 \mathrm{~min}$. A spectrophotometer (TECAN, Switzer-210 land) was used to measure the absorbances at a wavelength of $570 \mathrm{~nm}$. The cell inhibitory rate was calculated using the following equation: Cell inhibitory rate $=1-\left(\mathrm{OD}_{\text {treatment }}-\mathrm{OD}_{\text {blank }}\right) /\left(\mathrm{OD}_{\text {control }}-\mathrm{OD}_{\text {blank }}\right) \times$ $100 \%$. Each experiment was repeated at least three times.

\subsection{Determination of the apoptotic rate}

Apoptosis was quantified using an Annexin V-FITC apoptosis kit, which detects the surface exposure of phosphatidylserine in cells. Briefly, cells were seeded in 6-well plates at a density of $2 \times 10^{5}$ cells $/ \mathrm{mL}$ and incubated for $24 \mathrm{~h}$. Then, the cells were either treated with CIN only at doses of 0 , $6.25,12.5,25$, or $37.5 \mu \mathrm{g} / \mathrm{mL}$ for $48 \mathrm{~h}$ or with CIN and CUR $\left(\mathrm{CUR}_{4}\right.$ or $\left.\mathrm{CUR}_{8}\right)$ for $48 \mathrm{~h}$. The cells were harvested, washed with ice-cold phosphate buffer saline (PBS), resuspended in $300 \mu \mathrm{L}$ of binding buffer, and incubated with 5 $\mu \mathrm{L}$ of FITC-labeled Annexin V and $5 \mu \mathrm{L}$ of PI for $15 \mathrm{~min}$ at room temperature in the dark. Then, the samples were immediately analyzed using a flow cytometer (BD Inc, New York, USA) at an excitation wavelength of $488 \mathrm{~nm}$.

\subsection{Measurement of changes in the cytoskeleton and nucleus}

DAPI and FITC-phalloidin stains were used to observe the apoptotic cellular morphology and cytoskeletal rearrangements. Briefly, cells at a concentration of $3 \times 10^{5}$ cells $/ \mathrm{mL}$ were seeded in six-well plates and incubated for $24 \mathrm{~h}$. Af- 
terwards, the cells were treated with $\mathrm{CIN}_{25}$ or with a combination of $\mathrm{CIN}_{25}$ and CUR $\left(\mathrm{CUR}_{4}\right.$ or $\left.\mathrm{CUR}_{8}\right)$ for $24 \mathrm{~h}$. Then, the cells were fixed with $4 \%$ paraformaldehyde and stained successively with $50 \mu \mathrm{mol} / \mathrm{L}$ of DAPI for $5 \mathrm{~min}$ and $1 \mu \mathrm{mol} / \mathrm{L}$ of FITC-phalloidin for $60 \mathrm{~min}$ in the dark at room temperature. Finally, the cells were washed with PBS. A laser scanning confocal microscope (Carl Zeiss, Germany) was used to image the nuclear morphology and the organization of the F-actin cytoskeleton. The amounts of F-actin in HeLa cells that were untreated, treated solely with $\mathrm{CIN}_{25}$, or treated with a combination of $\mathrm{CIN}_{25}$ and CUR were determined by flow cytometry and actin-tracker green. Briefly, the cells, after being washed and harvested, were fixed by treatment with $4 \%$ paraformaldehyde for $15 \mathrm{~min}$. The cells were incubated with actin-tracker green for $30 \mathrm{~min}$, washed three times with PBS, and then suspended in PBS. The samples were immediately excited at $496 \mathrm{~nm}$ and analyzed with a flow cytometer (BD Inc, New York, USA).

\subsection{Atomic force microscopy}

Atomic force microscopy (AFM) was used to obtain topographic images of untreated cells, cells treated with $\mathrm{CIN}_{25}$, and with cells treated with a combination of $\mathrm{CIN}_{25}$ and CUR. Prior to use, the silicon nitride tips used in all AFM measurements were irradiated with ultraviolet light to remove organic contaminants. The curvature radius of the tips was less than $10 \mathrm{~nm}$, the elasticity coefficient was set at $2.8 \mathrm{~N} / \mathrm{m}$, and the oscillation frequency was set at $255 \mathrm{kHz}$. The samples were fixed by treatment with a $4 \%$ paraformaldehyde solution for 15 min after being monolayer-cultured, washed twice and dried at room temperature. Single-cell imaging experiments were performed on more than five cells, and each cell was scanned three times.

\subsection{Measurement of reactive oxygen species production}

Levels of intracellular reactive oxygen species (ROS) were determined by a Reactive Oxygen Species Assay Kit, which uses the staining probe DCFH-DA. DCFH-DA is a nonfluorescent probe that can freely penetrate the cell membrane. In cells, DCFH is deacetylated by esterases to form $2^{\prime}, 7^{\prime}$-dichlorofluorescin (DCFH), a non-fluorescent molecule that reacts in the presence of ROS to form the fluorescent product 2',7'-dichlorofluorescein (DCF). Briefly, cells were treated with $\mathrm{CIN}_{25}$ or with $\mathrm{CIN}_{25}$ in the presence of CUR $\left(\mathrm{CUR}_{4}\right.$ or $\left.\mathrm{CUR}_{8}\right)$ for $24 \mathrm{~h}$. The cells were then harvested, washed once with ice-cold $\mathrm{PBS}$, and incubated with $10 \mu \mathrm{mol} / \mathrm{L}$ of DCFH-DA at $37^{\circ} \mathrm{C}$ for $30 \mathrm{~min}$. After incubation with the fluorochrome, the cell culture was washed and resuspended in cold PBS. Then, to calculate the production of ROS, the fluorescence intensity was measured using flow cytometry; the flow cytometer was set at an excitation wavelength of $488 \mathrm{~nm}$ and an emission wavelength of $525 \mathrm{~nm}$.

\subsection{Detection of the intracellular free $\mathrm{Ca}^{2+}$ concentration}

The fluorescent dye Fluo-3 AM can cross the cell membrane and be converted into Fluo-3, which specifically reacts with $\mathrm{Ca}^{2+}$ to produce a molecule that strongly fluoresces. To detect changes in the free calcium level within the cytosol, both CIN-treated and untreated HeLa cells were incubated with Fluo-3 AM. The detailed procedure is as follows: HeLa cells that were untreated, treated with $\mathrm{CIN}_{25}$ for $24 \mathrm{~h}$, or treated with $\mathrm{CIN}_{25}$ in the presence of CUR $\left(\mathrm{CUR}_{4}\right.$ or $\left.\mathrm{CUR}_{8}\right)$ for $24 \mathrm{~h}$ were collected, washed twice in PBS, and resuspended in Fluo-3 AM $(5 \mu \mathrm{mol} / \mathrm{L})$ for $30 \mathrm{~min}$ in the dark. The intracellular $\mathrm{Ca}^{2+}$ concentration was measured using a flow cytometer set at an excitation wavelength of $488 \mathrm{~nm}$.

\subsection{Mitochondrial membrane potential measurement}

The MMP was monitored separately using two fluorescent dyes, rhodamin 123 and JC-1. The cells were harvested, washed, and resuspended in Rhodamine 123 ( $2 \mu \mathrm{mol} / \mathrm{L})$ for $30 \mathrm{~min}$ in the dark after being either treated with $\mathrm{CIN}_{25}$ for $24 \mathrm{~h}$ or a combination of $\mathrm{CIN}_{25}$ and CUR for 24 hours. The fluorescence was measured by flow cytometry at an excitation wavelength of $485 \mathrm{~nm}$.

In the normal cell, JC-1 accumulates and aggregates in the matrix of the mitochondria, forming J-aggregates and fluorescing bright red. In apoptotic cells, however, JC-1 cannot aggregate in the matrix of the mitochondria due to the reduction of the MMP. Instead, JC-1 remains in the cytoplasm in its monomeric form and fluoresces green. The cells were seeded in confocal petri dishes after a 24-h treatment with $\mathrm{CIN}_{25}$ or a 24-h treatment with $\mathrm{CIN}_{25}$ in combination with CUR $\left(\mathrm{CUR}_{4}\right.$ or $\left.\mathrm{CUR}_{8}\right)$. Then, $200 \mu \mathrm{L}$ of JC-1 staining solution was added. The cells were incubated in this solution for $15 \mathrm{~min}$. Then, the cells were washed with PBS and visualized directly under a laser scanning confocal microscope set to an excitation wavelength of 514 $\mathrm{nm}$.

\section{Results}

\subsection{The cytotoxic effect of CIN alone and in combination with CUR}

To determine the ability of CUR to enhance the CINinduced growth suppression of cells, cells were treated either with CIN alone or with CIN and CUR. CUR significantly enhances $\mathrm{CIN}$-induced cell cytotoxicity when the cells are incubated for $48 \mathrm{~h}$ at $37^{\circ} \mathrm{C}$. The results shown in Figure 1(a) revealed that after treatment for $48 \mathrm{~h}$, CIN alone exhibited dose-dependent inhibitory effects on the viability 

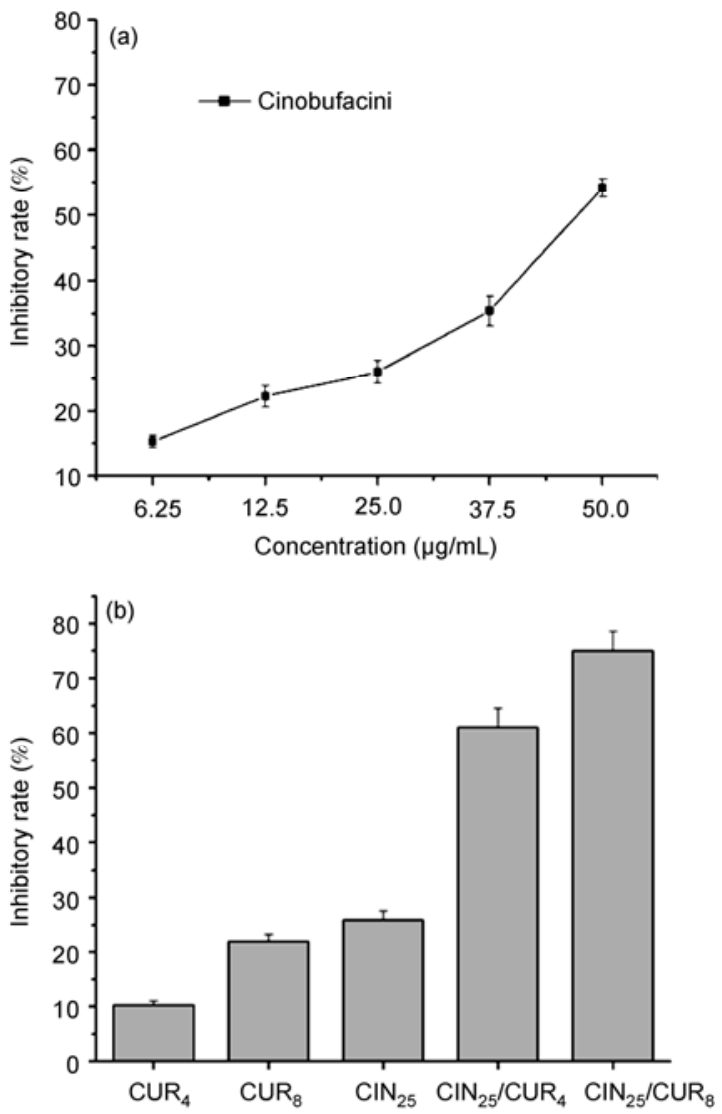

Figure 1 The cytotoxic effects of HeLa cells after a 48-h incubation with (a) CIN alone and (b) the combination of $\mathrm{CIN}_{25}$ and CUR as measured by MTT. of HeLa cells, and the inhibitory rate increased from $11 \%$ to $56 \%$. We adopted a CIN concentration of $25 \mu \mathrm{g} / \mathrm{mL}$ for the following combined experiments. The $\mathrm{CIN}_{25} / \mathrm{CUR}_{4}$ combination treatment showed a growth inhibition rate of $64 \%$, which is 2.5 times higher than that of $\mathrm{CIN}_{25}$ alone. The $\mathrm{CIN}_{25} / \mathrm{CUR}_{8}$ combination treatment showed a growth inhibition rate of $75 \%$, which is 3 times higher than that of $\mathrm{CIN}_{25}$ alone (Figure 1(b)). These data suggested that the CIN-induced growth suppression of HeLa cells could be strengthened in the presence of CUR.

\subsection{Apoptosis of HeLa cells induced by CIN alone and in combination with CUR}

Annexin V-FITC/PI staining was carried out to assess the extent and mode of HeLa cell death. The kit measures phosphatidylserine turnover from the inner to the outer lipid layer of the plasma membrane, an event typically associated with apoptosis. The proportions of early apoptotic cells were $8.05 \%, 10.62 \%, 15.43 \%$, and $18.24 \%$ after HeLa cells were treated with CIN at concentrations of $6.25,12.5,25$, 37.5 and $50 \mu \mathrm{g} / \mathrm{mL}$, respectively (Figure 2(a)-(c)). Furthermore, Figure 2(d)-(f) reveal that compared with the $\mathrm{CIN}_{25}$ only treatment, the combined treatment significantly enhances the proportion of early apoptotic cells. The early apoptotic rates were $31.9 \%$ and $49.2 \%$ for $\mathrm{CIN}_{25} / \mathrm{CUR}_{4}$ and $\mathrm{CIN}_{25} / \mathrm{CUR}_{8}$, respectively. In contrast, the percentage of secondary necrosis, as measured by staining with both Annexin V/FITC and PI, did not significantly change.
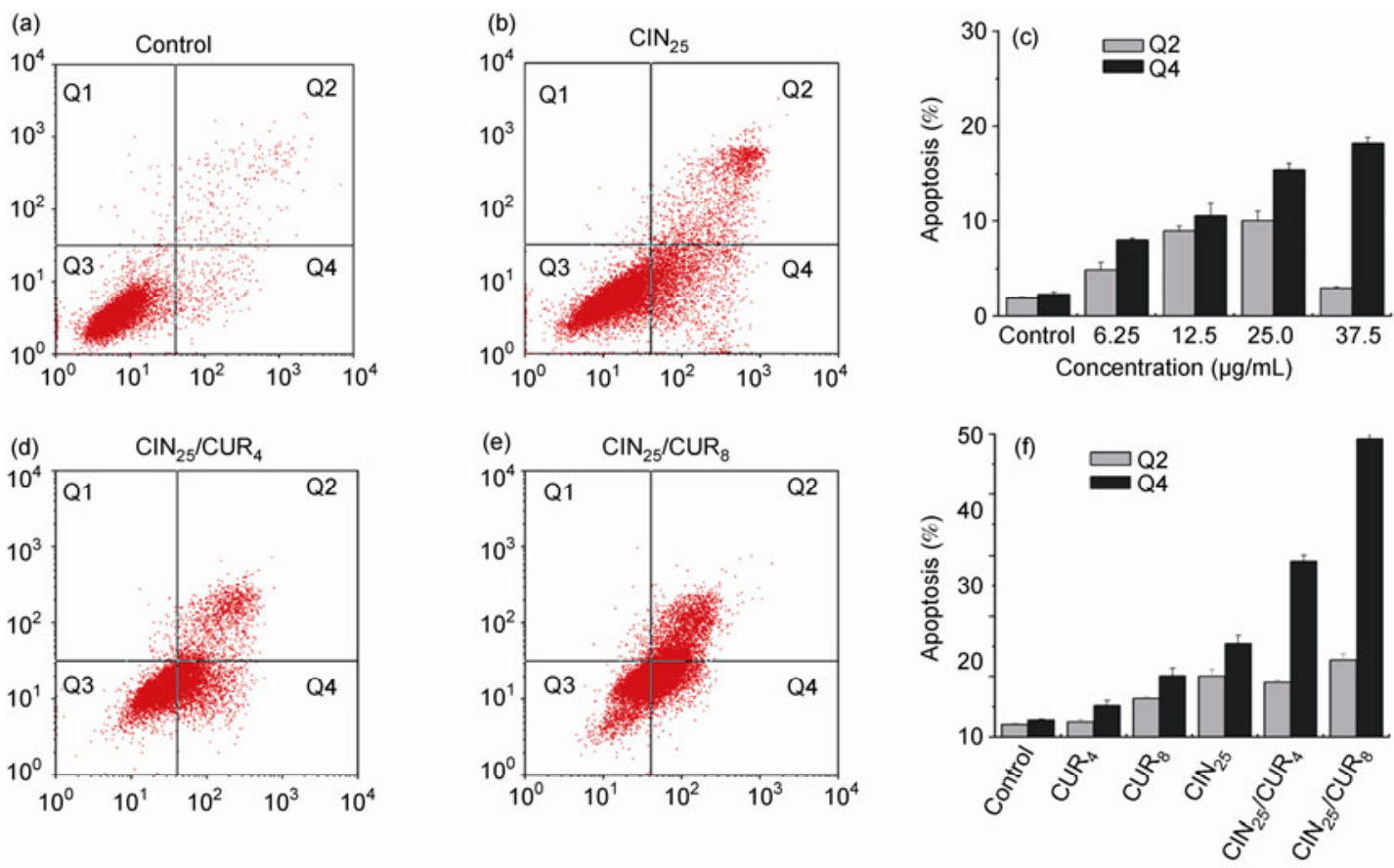

Figure 2 (Color online) Effect of CIN alone or combined with CUR on apoptosis as determined by flow cytometry. (a), (b) The representative apoptosis induced by CIN only. (d), (e) The representative apoptosis induced by the combination of $\mathrm{CIN}_{25}$ and CUR (CUR 4 or CUR 8 ). (c), (f) The statistical data for $((\mathrm{a}),(\mathrm{b}))$ and $((\mathrm{d}),(\mathrm{e}))$ respectively. 


\subsection{Derangement of the cytoskeleton and damage to the nucleus}

Changes to cellular morphology were examined by specific staining after treatment with CIN alone or in combination with CUR. Specifically, the F-actin cytoskeleton was stained with Rhodamine-labeled phalloidin, and the nucleus was stained with DAPI. The cells were examined using a laser scanning confocal microscope (LSCM). Previous reports provided evidence that cytoskeletal alterations could induce apoptosis as well as necrosis in a variety of models [22-26]. Thus, it was very important to investigate whether the combined treatment affected the arrangement of the cytoskeleton. In Figure 3(a) and (b), stains of control cells showed a generally homogeneous distribution of F-actin in the cytoplasm and intact, plump and uniform nuclei. After the 24-h treatment with $\mathrm{CIN}_{25}$, the F-actin aggregated and formed dot-like structures, and the nuclei presented as hippocrepiform. Furthermore, the cytoskeletal networks completely disappeared. When treated with the $\mathrm{CIN}_{25} / \mathrm{CUR}_{8}$ combination, the cells presented the typical morphology of apoptotic cells: The nuclei had broken, the chromatin was condensed and gathered at the periphery of the nuclear membrane, and the edges of the cells became blurred. Additionally, the cells appeared more widely distributed on the substrate. These observations indicate that the combination treatment significantly inhibited the proliferation of HeLa cells by changing the organization of the F-actin cytoskeleton and breaking the nucleus. Actin-tracker green was also employed to measure the amount of F-actin before and after treatment with $\mathrm{CIN}_{25}$ only or treatment with $\mathrm{CIN}_{25}$ and CUR $\left(\mathrm{CUR}_{4}\right.$ or $\left.\mathrm{CUR}_{8}\right)$. Figure 3(c) shows that the fluorescent signal of the actin tracker in HeLa cells decreased more in the combined treatment than in the treatment with $\mathrm{CIN}_{25}$ alone. The MFI of cells treated with $\mathrm{CIN}_{25}$ alone was 14670 , while the MFI of cells treated with the $\mathrm{CIN}_{25} / \mathrm{CUR}_{8}$ combination decreased to 9339 . Taken together, these results revealed that the combined treatment was able to alter the organization of the cytoskeleton architecture by disrupting F-actin distribution and decreasing the expression of F-actin in HeLa cells more easily than the treatment with $\mathrm{CIN}_{25}$ alone.

\subsection{Cell morphology and cell membrane ultrastructure characterization}

As a nondestructive cell surface imaging tool, AFM was used to observe a variety of changes in the surface morphology and membrane ultrastructure of HeLa cells treated with CIN in the presence and absence of CUR [27]. As (a)
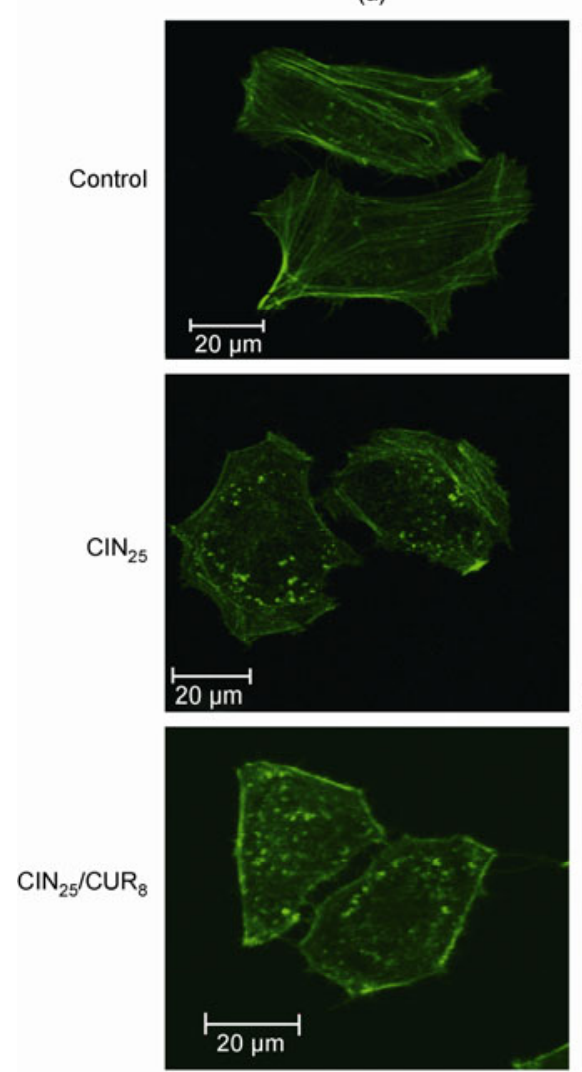

(b)
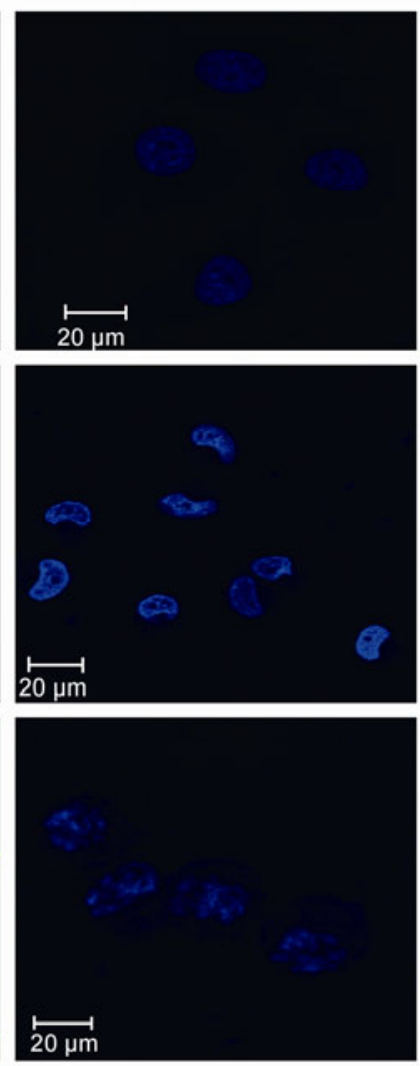

(c)
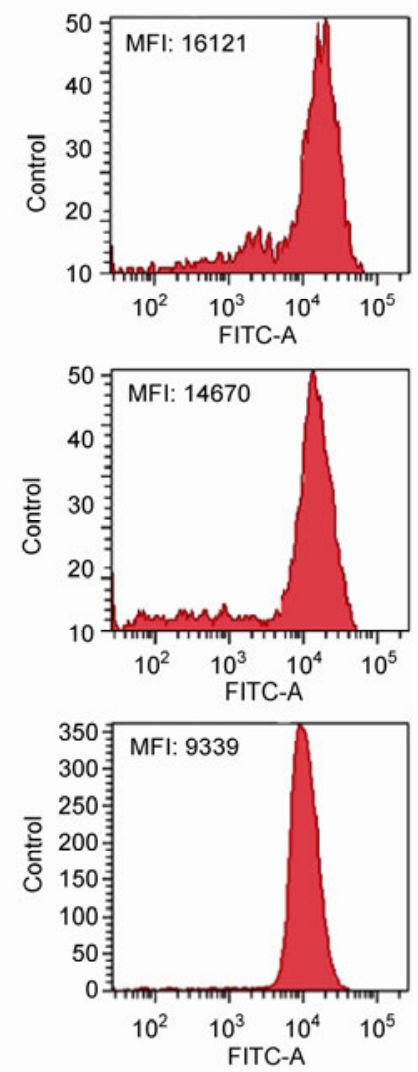

Figure 3 (Color online) Changes in the F-actin cytoskeleton and nucleus after treatment with $\mathrm{CIN}_{25}$ alone or in combination with CUR 8 . (a) The morphology of cytoskeleton F-actin changes. (b) Variation in the morphology of the nucleus. (c) Alterations to F-actin expression. 


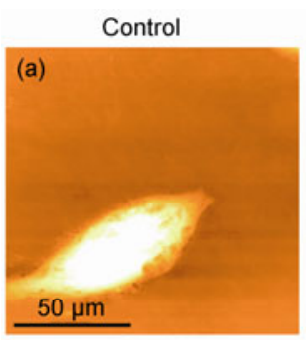

$\mathrm{CIN}_{25} / \mathrm{CUR}_{4}$

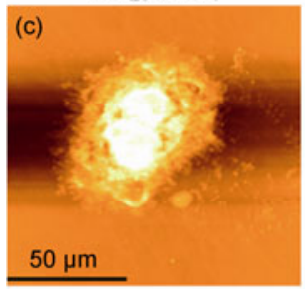

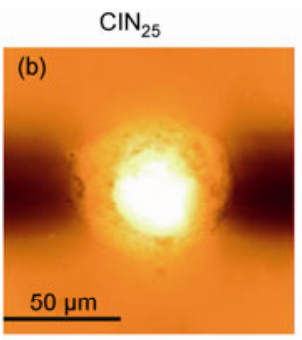

$\mathrm{CIN}_{25} / \mathrm{CUR}_{8}$

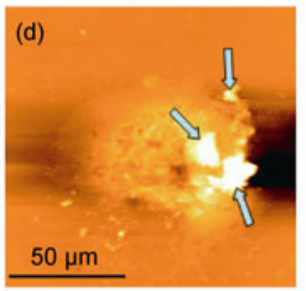

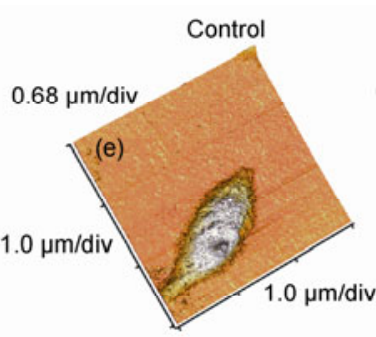
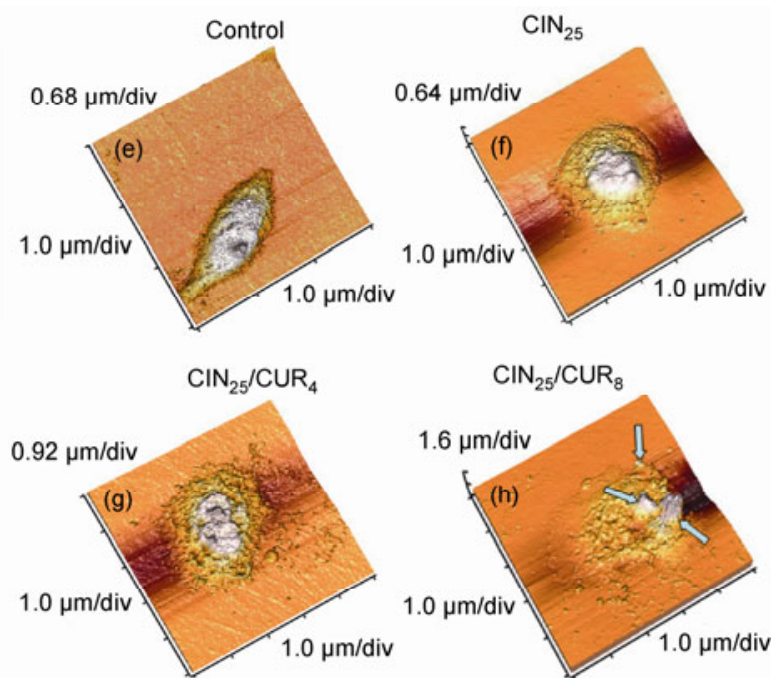

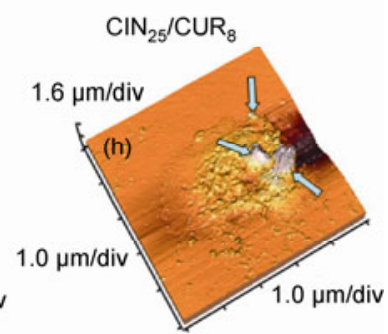

Figure 4 (Color online) AFM topographic data of HeLa cells. (a)-(d) The topography of the control group, CIN 25 group, $\mathrm{CIN}_{25} / \mathrm{CUR}_{4}$ group and $\mathrm{CIN}_{25} / \mathrm{CUR}_{8}$ group, respectively. (e)-(h) The 3-D modes corresponding to (a)-(d).

shown in Figure 4, the control cells had a regular, long spindle-shaped morphology, and the nuclei were plump and elliptical. The cell tails were unrolled, and the cell surface was relatively smooth and intact (Figure 4(a) and (e)). After treatment with $\mathrm{CIN}_{25}$ alone, the cells shrunk, but the nuclei remained intact (Figure 2(b) and (f)). However, as shown in Figure $4(\mathrm{c})$ and $(\mathrm{g})$, when the cells were also treated with the lower CUR concentration $\left(\mathrm{CIN}_{25} / \mathrm{CUR}_{4}\right)$, the cells were deformed and shrunken. The edges of the cells became blurred, and the nuclei were no longer uniform. Upon increasing the CUR concentration to $8 \mu \mathrm{g} / \mathrm{mL}\left(\mathrm{CIN}_{25} / \mathrm{CUR}_{8}\right)$ in the combined system, the cells varied significantly in size and shape. For example, cells collapsed, and the cytoplasm of many cells leaked. Additionally, blebbing pores and apoptotic bodies appeared over the cell surface (Figure 4(d) and (h)). These characteristics are indicative of apoptosis or necrosis.

Moreover, AFM can provide nanoscale insight into ultrastructural changes occurring in the topography of the cell membrane [28]. Figure 5 shows the cell surface ultrastructure of HeLa cells treated with CIN alone or in combination with CUR. The cell membrane architecture of the control cells (Figure 5 (a)) was homogeneous and presented a granular morphology with a surface particle size of approximately $45 \mathrm{~nm}$. After $\mathrm{CIN}_{25}$ treatment alone, the surface of the cells became rough, and the nanoparticle size increased to $70 \mathrm{~nm}$; the form became scattered. The visibly protruding particles are clusters of membrane proteins [29]. Furthermore, the cytoskeletal structure of the control cells presented regular networks of F-actin, while the cytoskeleton networks disappeared and presented a gathered state after the combined treatments (shown in Figure 3). The nanoparticles on the cell membrane were aggregated and not a uniform size. The size increased to 150 and $190 \mathrm{~nm}$ for the $\mathrm{CIN}_{25}$ / $\mathrm{CUR}_{4}$ and $\mathrm{CIN}_{25} / \mathrm{CUR}_{8}$ combinations, respectively (Figure 5(c) and (d)). Taken together, these morphological data revealed dose-dependent morphological changes corresponding to the combined treatment. The combined treatment mainly induced cell apoptosis. The morphological changes implied that some physiological changes could occur in membrane proteins, including the opening and closing of ion channels, the disruption of the ion channel structure, and alterations to the chemical composition of the outer membrane [30]. The altered chemical composition of the outer membrane may have led to changes in the ultrastructure of the cytoskeleton and membrane surface. Further, these results provide morphological information about the combined effects of induced HeLa cell toxicity (damage). The combined treatments affected the external structure of HeLa cells. This result might be related to F-actin disorganization, mitochondria-dependent apoptosis, or disruption of the nucleus.

\subsection{Mitochondria-dependent apoptosis induced by enhancement of ROS overproduction and the free $\mathrm{Ca}^{2+}$ level}

Previous studies have shown that ROS, by-products of aerobic metabolism, play vital roles in intracellular signaling cascades $[31,32]$. Mitochondria are one of the major sources of intracellular ROS, and overproduction of ROS can trigger stress pathways that ultimately lead to cell apoptosis [33]. High ROS levels can also cause cellular damage, depending on the duration of ROS stress [34], and ROS can function as anti-tumor agents [35]. A novel cancer therapy strategy preferentially eradicates cancer cells by targeting the ROS stress-response pathway [36]. Thus, we investigated changes in ROS production in cells before and after being treated with CIN or CIN in combination with CUR. Figure 6(a) and (b) show the data collected by the flow 
(a)

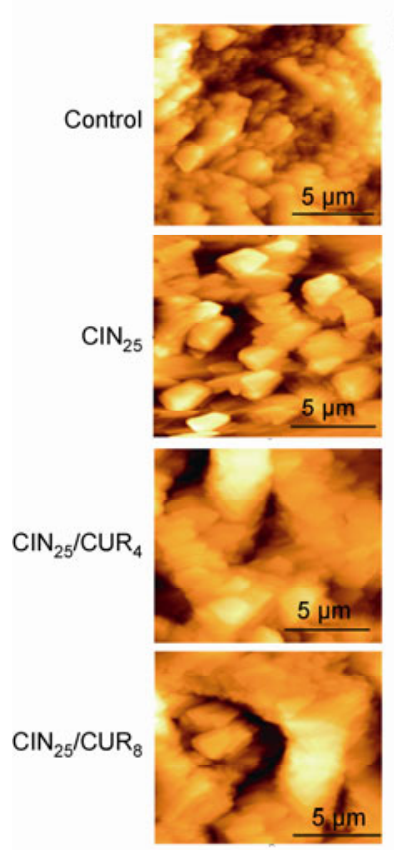

(b)
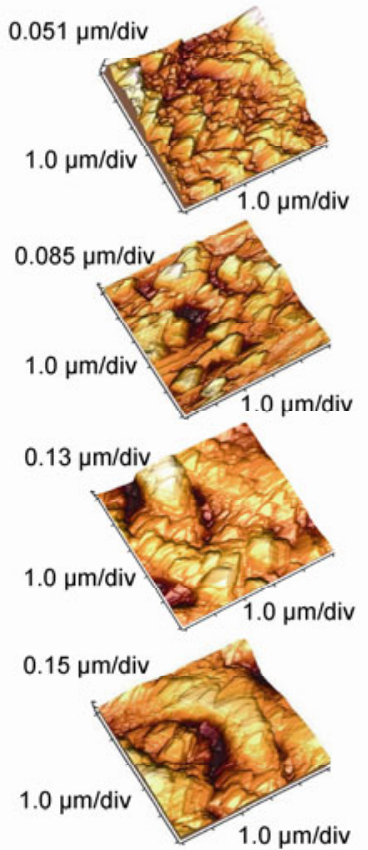

(c)
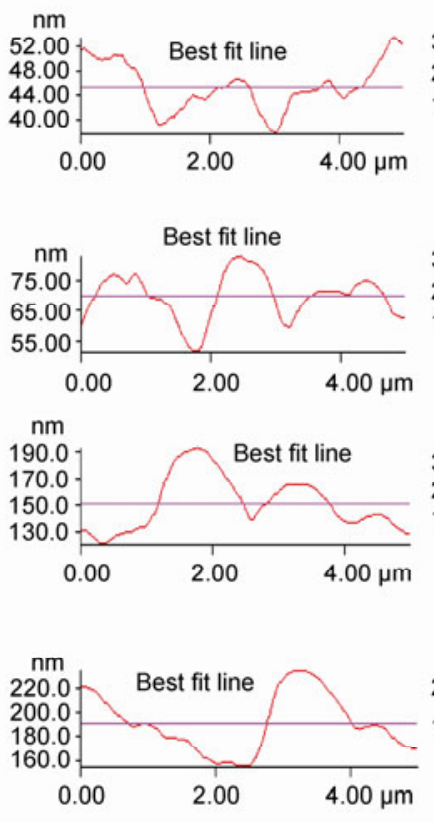

(d)
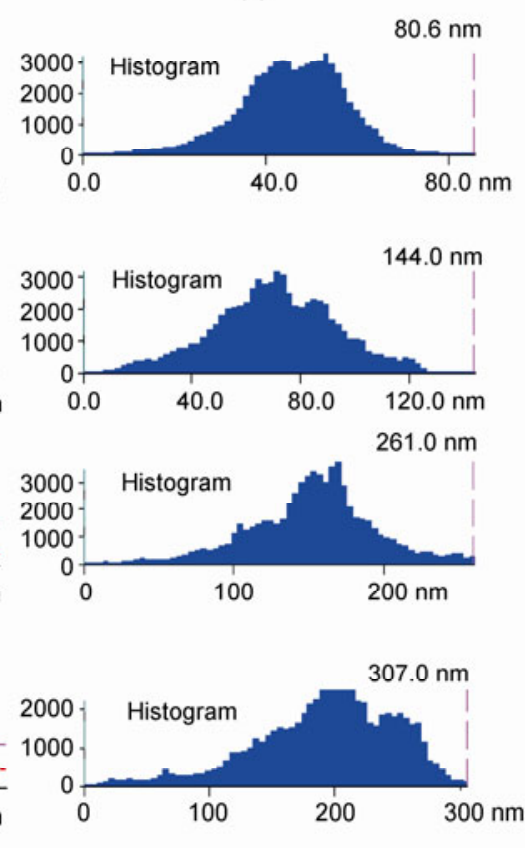

Figure 5 (Color online) AFM ultrastructural data of $\mathrm{HeLa}$ cells before and after treatment with $\mathrm{CIN}_{25}$ only or treatment with $\mathrm{CIN}_{25}$ in combination with CUR $\left(\mathrm{CUR}_{4}\right.$ or $\mathrm{CUR}{ }_{8}$ ). (a) The topography mode $(3 \mu \mathrm{m} \times 3 \mu \mathrm{m})$. (b) The related 3-D images corresponding to (a). (c) The height profiles. (d) The grain diameter histograms of the cell surface extracted from related images (a).
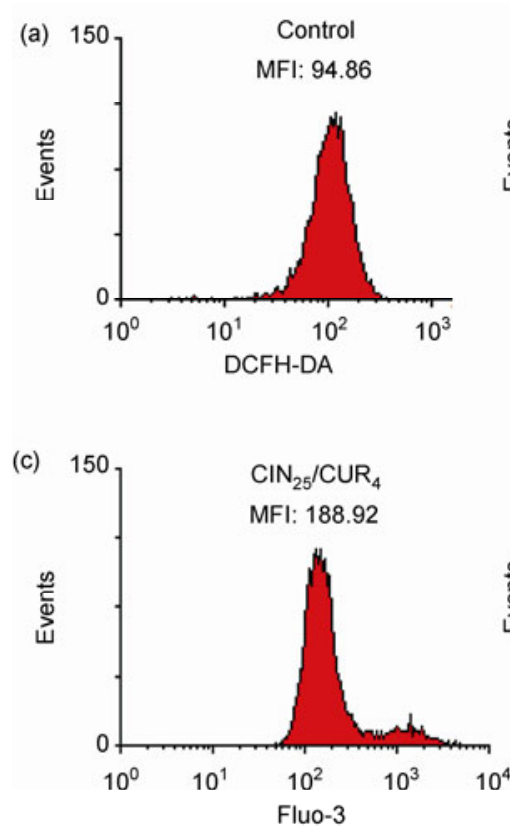
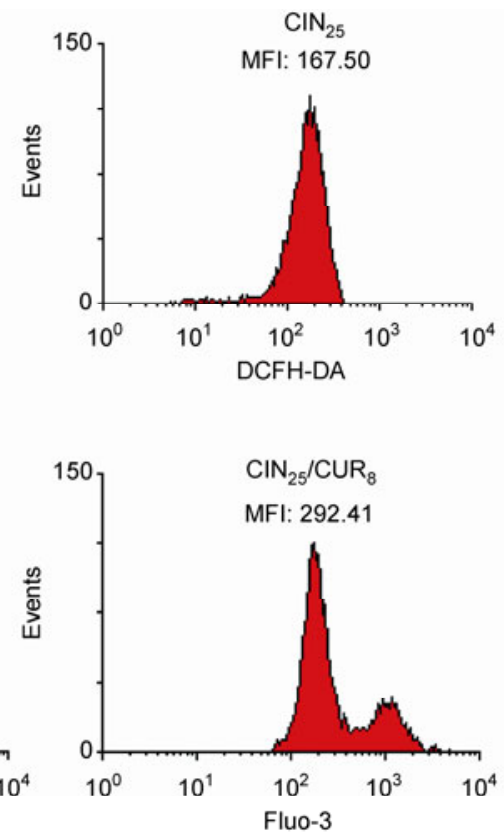
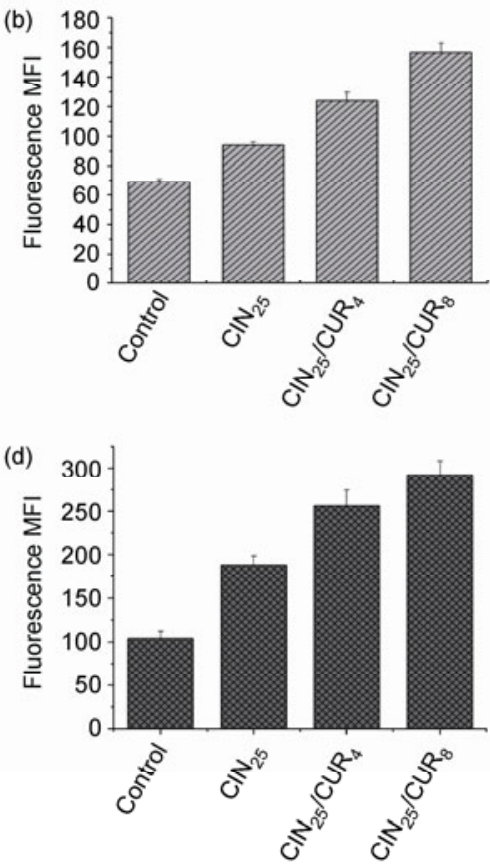

Figure 6 (Color online) Flow cytometric analysis of ROS and intracellular free calcium $\left(\mathrm{Ca}^{2+}\right)$ in HeLa cells treated with CIN only or CIN in combination with CUR. (a) Typical change of ROS determined by a DCFH-DA detection Kit. (b) Changes in ROS levels in HeLa cells treated with $\mathrm{CIN}_{25}$ for $24 \mathrm{~h}$ or with $\mathrm{CIN}_{25}$ and CUR (CUR 4 or $\mathrm{CUR}_{8}$ ) for $24 \mathrm{~h}$. (c) Typical changes in $\mathrm{Ca}^{2+}$ levels determined by a Fluo-3 Detection Kit. (d) Changes in Ca ${ }^{2+}$ levels in HeLa cells treated solely with $\mathrm{CIN}_{25}$ for $24 \mathrm{~h}$ or with $\mathrm{CIN}_{25}$ and CUR $\left(\mathrm{CUR}_{4}\right.$ or $\left.\mathrm{CUR}_{8}\right)$ for $24 \mathrm{~h}$.

cytometer and the mean fluorescence intensity (MFI), as dictated by ROS overproduction. $\mathrm{CIN}_{25}$ alone only slightly elevated the intracellular ROS levels: the MFI for $\mathrm{CIN}_{25}$ treatment alone was 94.86, while the MFI for the control group was 69.20. Furthermore, the combination treatment yielded a significant increase in ROS production. The MFI values were 128.20 and 167.50 for the combined $\mathrm{CIN}_{25} /$ $\mathrm{CUR}_{4}$ and $\mathrm{CIN}_{25} / \mathrm{CUR}_{8}$ treatments, respectively (Figure 6(a) 
and (b)). We have demonstrated that the combination treatment triggers oxidative stress and that oxidative stress plays a major role in inducing apoptotic changes in HeLa cells.

Increased intracellular $\mathrm{Ca}^{2+}$ concentration is also recognized as an important marker of cell death and injury [37]. Thus, we measured the intensity of fluo- 3 fluorescence, an indicator of the $\mathrm{Ca}^{2+}$ concentration. Figure 6(c) and (d) showed that the MFI of intracellular free $\mathrm{Ca}^{2+}$ was 109.60 for the control group and 188.92 when the cells were treated with $\mathrm{CIN}_{25}$ alone. After CUR treatment, the cells displayed much higher $\mathrm{Ca}^{2+}$ concentrations; the MFI values were 261.9 and 292.41 for the $\mathrm{CIN}_{25} / \mathrm{CUR}_{4}$ and $\mathrm{CIN}_{25} / \mathrm{CUR}_{8}$ combination treatments, respectively. The results indicated that the increase in intracellular $\mathrm{Ca}^{2+}$ concentration was related to HeLa cell apoptosis. Cell signaling can be activated by a temporary increase in the cytoplasmic calcium level as a result of a calcium channel opening in the plasma membrane or the endoplasmic reticulum [38]. The rise in the cytoplasmic $\mathrm{Ca}^{2+}$ concentration resulting from the combined treatment might be largely associated with membrane collapse (Figure 4). Membrane collapse could promote the opening of $\mathrm{Ca}^{2+}$ channels in the cell membrane and the subsequent influx of $\mathrm{Ca}^{2+}$.

\subsection{MMP loss induced by enhancement of ROS over- production}

The loss of MMP could lead to cytochrome c release $[39,40]$, a decrease in adenosine triphosphate (ATP) genera- tion [41], the activation of caspases and initiation of apoptotic cascades [42], all of which play important roles in the induction of cell death (necrosis or apoptosis). JC-1 staining was used to qualitatively confirm MMP disruption [43]. Rhodamine 123 was used to quantitatively detect the loss of MMP [44]. The JC-1 stain provides a distinct visual result, as red fluorescence is the indicator of higher MMP levels, while green fluorescence is the indicator of lower MMP levels. The combined treatment induced a dose-dependent fluorescent shift from red to green, indicating that the combined treatment disrupted MMP in HeLa cells (Figure 7(a)). Moreover, the fluorescent intensity of Rhodamine 123 was reduced more by the combined treatment than by treatment with $\mathrm{CIN}_{25}$ alone. Figure 7(b) and (c) show that the MFI of the MMP was 103.10 for the control group and that the MFI of the MMP decreased to 83.62 upon treatment with $\mathrm{CIN}_{25}$ alone. After CUR treatment, the MFI values were reduced to 38.46 and 16.68 for the combined $\mathrm{CIN}_{25} / \mathrm{CUR}_{4}$ and $\mathrm{CIN}_{25} / \mathrm{CUR}_{8}$ treatments, respectively. This information demonstrated that the combined treatment induces MMP disruption much more effectively than $\mathrm{CIN}_{25}$ treatment alone. The loss of MMP should play an important role in the apoptosis of HeLa cells.

\section{Discussion}

In this study, we first assessed the combined effects of CUR and CIN on human cervical carcinoma cells. The data show (a)

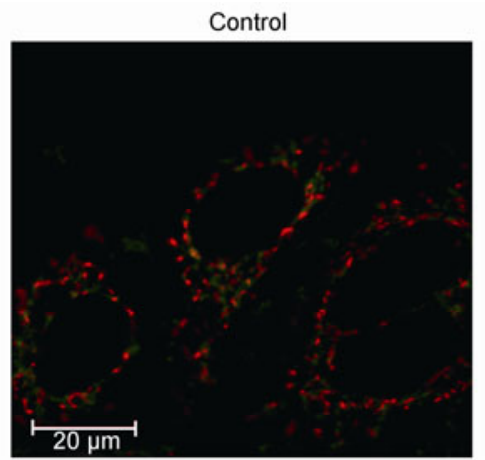

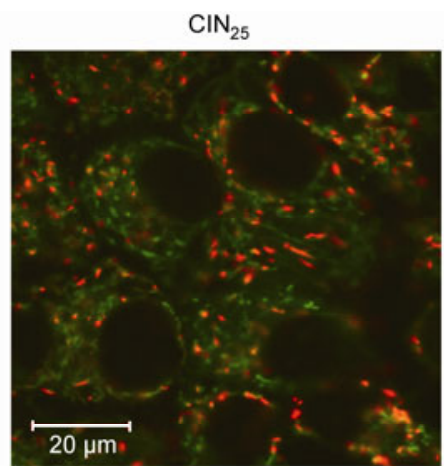

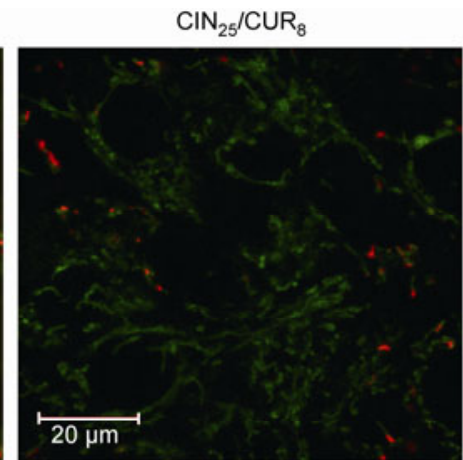

(b)
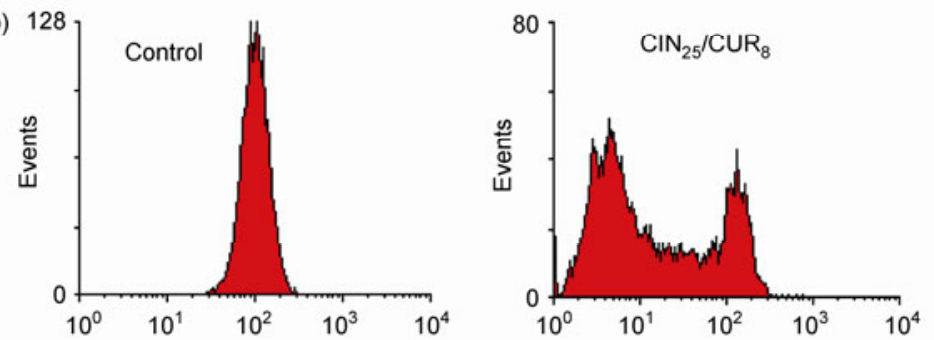

(c)

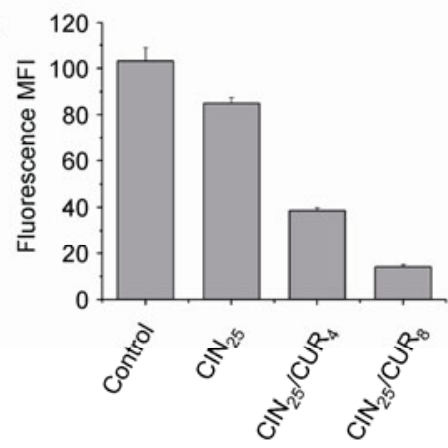

Figure 7 (Color online) The changes of membrane potential in HeLa cells treated with CIN alone or in combination with CUR. (a) LSCM images of changes to the MMP in the $\mathrm{CIN}_{25}$ only or combined treatment of HeLa cells stained with JC-1. (b) Flow cytometric analysis of typical changes to MMP determined by Rhodamine 123 staining. (c) Statistical data of changes to the MMP in HeLa cells treated for $24 \mathrm{~h}$ with CIN ${ }_{25}$ alone or in combination with CUR. 
that inhibition of viability and induction of apoptosis are more significant for the combined treatment than for treatment with CIN alone.

Moreover, the disorganization and depolymerization of the F-actin skeleton and the damage to the nucleus show that combination treatments induce apoptosis. The intracellular oxidative stress resulting from the accumulation of ROS plays a key role in inducing apoptosis [45,46]. The increase of intracellular ROS by CIN is considered to be a major mechanism in CIN-induced cell injury and the principal CIN treatment in cancer therapy [47]. ROS lead to free radical attack of membrane phospholipids and the loss of MMP, which releases inter-membrane proteins (such as cytochrome $c$ ) from the mitochondria. This process leads to the opening of a mitochondrial permeability transition pore and $\mathrm{Ca}^{2+}$ efflux from the cytoplasm $[48,49]$. In the presence of CUR, the increased ROS level suggests that the combined treatment triggers the generation of oxidative stress. CUR could be a suitable chemical sensitizer of CIN-induced apoptosis. The MMP governs the electrochemical forces employed for ATP synthesis and other metabolic activities, including the maintenance of $\mathrm{Ca}^{2+}$ homeostasis within the mitochondria [50]. Due to the energy requirements of the different $\mathrm{Ca}^{2+}$ transport systems that control $\mathrm{Ca}^{2+}$ homestasis, the loss of MMP and the subsequent ATP depletion invariably result in the disruption of intracellular $\mathrm{Ca}^{2+}$ homeostasis. These results demonstrate that the CUR enhancement of CIN-induced apoptosis in HeLa cells operates via ROS-dependent pathways. In this process, the ROS level and cytosolic free $\mathrm{Ca}^{2+}$ concentration increase, and the MMP decreases more significantly than after CIN treatment alone.

Changes to the membrane structure have a direct influence on cellular function [51,52]. AFM morphological data indicate that the cell surface morphology changed from homogeneous to heterogenous. The AFM morphological data indicated shrinkage, blebbing, and the appearance of pores on the membrane. Furthermore, the ultrastructure of control cells presented regular networks of F-actin (shown in Figure 3), The cytoskeleton networks disappeared and presented a gathered state after the combined treatment $\left(\mathrm{CIN}_{25} / \mathrm{CUR}_{8}\right.$ ) (Figure 3 ). $\mathrm{CIN}_{25}$-induced cells had a particle size of $70 \mathrm{~nm}$. The particle size on the cell surface increased to $150 \mathrm{~nm}$ for $\mathrm{CIN}_{25} / \mathrm{CUR}_{4}$ and to $190 \mathrm{~nm}$ for $\mathrm{CIN}_{25} / \mathrm{CUR}_{8}$ (Figure 5). This result suggested the altered chemical composition of the outer membrane or a reduction in the number of microvilli upon combined treatment with CIN and CUR.

Furthermore, AFM enabled the detection of subtle (nanoscale) changes to the structure of the cancer cell upon treatment with $\mathrm{CIN}_{25}$ alone or in combination with CUR. These results provided insight into the structure-function relationship of cell surfaces and yielded a more comprehensive understanding of the drug-cell interactions during chemotherapy. These treatments disrupted the MMP and subsequently induced apoptosis. Moreover, these treatments were reflected in the ultrastructure of the cell surface.

\section{Conclusion}

This research provided detailed insights into potentially novel cervical carcinoma treatments. Moreover, this study demonstrated CUR's enhancement of CIN-induced apoptosis. The results from this study could provide visual diagnoses to early stage apoptosis in tumor cells in response to anti-cancer drugs. These studies also yield insight into drugcell interactions. However, more research will be required to fully understand the specific anti-cancer mechanism of the enhancement of CIN-induced apoptosis by CUR.

This work was supported by the National Basic Research Program of China (2010CB833603), Overseas, Hong Kong \& Macao Cooperative Research Funds of China (31129002), the National Natural Science Foundation of China (30872404), Jinan University's Scientific Research Cultivation and Innovation Fund (21612601).

1 Bonomi P, Blessing J A, Stehman F B, et al. Randomized trial of three cisplatin dose schedules in squamous-cell carcinoma of the cervix: A Gynecologic Oncology Group study. J Clin Oncol, 1985, 3: 1079-1085

2 Omura G A. Chemotherapy for cervix cancer. Semin Oncol, 1994, 21 54-62

3 McGuire W P, Blessing J A, Moore D, et al. Paclitaxel has moderate activity in squamous cervix cancer. A Gynecologic Oncology Group study. J Clin Oncol, 1996, 14: 792-795

4 Sutton G P, Blessing J A, McGuire W P, et al. Phase II trial of ifosfamide and mesna in patients with advanced or recurrent squamous carcinoma of the cervix who had never received chemotherapy: A Gynecologic Oncology Group study. Am J Obstet Gynecol, 1993, 168: 805-807

5 Muderspach L I, Blessing J A, Levenback C, et al. A phase II study of topotecan in patients with squamous cell carcinoma of the cervix: A Gynecologic Oncology Group study. Gynecol Oncol, 2001, 81: 213-215

6 Long III H J, Bundy B N, Grendys E C, et al. Randomized phase III trial of cisplatin with orwithout topotecan in carcinoma of the uterine cervix: A Gynecologic Oncology Group study. J Clin Oncol, 2005, 23: 4626-4633

7 Luk J M, Wang X, Liu P, et al. Traditional Chinese herbal medicines for treatment of liver fibrosis and cancer: From laboratory discovery to clinical evaluation. Liver Int, 2007, 27: 879-890

8 Zuo X D, Cui Y A, Qin S K, et al. Clinical research progress on the antitumor effects of cinobufacini (in Chinese). Chin Clin Oncol, 2003, 8: 232-235

9 Zuo X D, Cui Y A, Qin S K, et al. Effect of cinobufacini on tumor cell cycle and expression of Bcl-2 protein (in Chinese). Mod J Integr Chin Tradit West Med, 2003, 12: 567-568

10 Deshpande S S, Maru G B. Effects of curcumin on the formation of benzo [a] pyrene derived DNA adducts in vitro. Cancer Lett, 1995, 96: 71-80

11 Sharma R A, Gescher A J, Steward W P. Curcumin: The story so far. Eur J Cancer, 2005, 41: 1955-1968

12 Maheshwari R K, Singh A K, Gaddipati J, et al. Multiple biological activities of curcumin: A short review. Life Sci, 2006, 78: 2081-2087

13 Ono K, Hasegawa K, Naike H, et al. Curcumin has potent anti-amyloidogenic effects for Alzheimer's beta-amyloidfibrils in vitro. Neurosci Res, 2004, 75: 742-750

14 Aggarwal B B, Kumar A, Bharti A C. Anticancer potential of curcu- 
min: Preclinical and clinical studies. Anticancer Res, 2003, 23: 363398

15 Syng-ai C, Kumari A L, Khar A. Effect of curcumin on normal and tumor cells: Role of glutathione and Bcl-2. Mol Cancer Ther, 2004, 3: 1101-1108

16 Kunwar A, Barik A, Mishra B, et al. Quantitative cellular uptake, localization and cytotoxicitof curcumin in normal and tumor cells. Biochim Biophys Acta-Gen Subj, 2008, 1780: 673-679

17 Scarlett J L, Sheard P W, Hughes G, et al. Changes in mitochondrial membrane potential during staurosporine-induced apoptosis in Jurkat cells. FEBS Lett, 2000, 475: 267-272

18 Puech P H, Poole K, Knebel D, et al. A new technical approach to quantify cell-cell adhesion forces by AFM. Ultramicroscopy, 2006, 106: 637-644

19 Alarmo E, Pärssinen J, Ketolainen J M, et al. BMP7 influences proliferation, migration, and invasion of breast cancer cells. Cancer Lett, 2009, 275: 35-43

20 Heidemann S R, Wirtz D. Towards a regional approach to cellmechanics. Trends Cell Biol, 2004, 14: 160-166

21 Suresh S. Biomechanics and biophysics of cancer cells. Acta Mater, 2007, 55: 3989-4014

22 Shimizu S, Eguchi Y, Kamiike W. Induction of apoptosis as well as necrosis by hypoxia and predominant prevention of apoptosis by Bcl-2 and Bcl-XL. Cancer Res, 1996, 56: 2161-2166

23 Grzanka A, Grzanka D, Orlikowska M. Cytoskeletal reorganization during process of apoptosis induced by cytostatic drugs in K-562 and HL-60 leukemia cell lines. Biochem Pharmacol, 2003, 66: 16111617

24 Melanie H, Amanda M G V, Pieter S, et al. Rho-kinase-dependent F-actin rearrangement in involved in the inhibition of PI3-kinase/Akt during ischemia-reperfusion-induced endothelial cell apoptosis. Apoptosis, 2008, 13: 404-412

25 Olivia N T, Jocelyne H, Jacqueline B. Cytoskeleton and apoptosis. Biochem Pharmacol, 2008, 76: 11-18

26 Escobar M L, Echeverría R O, Vázquez-Nin G H. Combined apoptosis and autophagy, the process that eliminates the oocytes of atretic follicles in immature rats. Apoptosis, 2008, 13: 1253-1266

27 Lekka M, Laidler P. Applicability of AFM in cancer detection. Nat Nanotechnol, 2009, 4: 72-73

28 Jin H, Huang X, Chen Y, et al. Photoinactivation effects of hematoporphyrin monomethyl ether on Gram-positive and -negative bacteria detected by atomic force microscopy. Appl Microbiol Biotechnol, 2010, 88: 761-770

29 Christian L G, Lesniewska E, Giocondi M, et al. Imaging of the surface of living cells by low-force contact-mode atomic force microscopy. Biophys J, 1998, 75: 695-703

30 Wang M, Ruan Y X, Chen Q, et al. Curcumin induced HepG2 cell apoptosis-associated mitochondrial membrane potential and intracellular free $\mathrm{Ca}^{2+}$ concentration. Eur J Pharmacol, 2011, 650: 41-47

31 Denning T L, Takaishi H, Crowe S E, et al. Oxidative stress induces the expression of Fas and Fas ligand and apoptosis in murine intestinal epithelial cell. Free Radic Biol Med, 2002, 33: 1641-1650

32 Lin Y, Choksi S, Shen H M, et al. Tumor necrosis factor-induced nonapoptotic cell death requires receptor-interacting protein-mediated cellular reactive oxygen species accumulation. J Biol Chem, 2004, 279: 10822-10828

33 Ortiz-Sánchez E, Daniels T R, Helguera G, et al. Enhanced cytotoxicity of an anti-transferrin receptor IgG3-avidin fusion protein in combination with gambogic acid against human malignant hemato- poietic cells: Functional relevance of iron, the receptor, and reactive oxygen species. Leukemia, 2009, 23: 59-70

34 Trachootham D, Zhou Y, Zhang H, et al. Selective killing of oncogenically transformed cells through a ROS-mediated mechanism by $\beta$-phenylethyl isothiocyanate. Cancer Cell, 2006, 10: 241-252

35 Lu M, Bi C S, Gong X G, et al. Anti-proliferative effects of recombinant iron superoxide dismutase on HepG2 cells via a redoxdependent PI3k/Akt pathway. Appl Microbiol Biotechnol, 2007, 76: 193-201

36 Raj L, Ide T, Gurkar A U, et al. Selective killing of cancer cells by a small molecule targeting the stress response to ROS. Nature, 2011, 475: 231-234

37 Orrenius S, Zhivotovsky B, Nicotera P. Regulation of cell death: The calcium-apoptosis link. Nat Rev Mol Cell Biol, 2003, 4: 552-565

38 Jared L S, Philip W S, Gillian H, et al. Changes in mitochondrial membrane potential during staurosporine-induced apoptosis in Jurkat cells. FEBS Lett, 2000, 475: 267-272

39 Douglas R G, Guido K. The pathophysiology of mitochondrial cell death. Science, 2004, 305: 626-629

40 Waterhouse N J, Goldstein J C, Ahsen O V, et al. Cytochrome $c$ maintains mitochondrial transmem-brane potential and ATP generation after outer mitochondrial membrane perme-abilization during the apoptotic process. J Cell Biol, 2001, 153: 319-328

$41 \mathrm{Hu}$ W, Kavanagh J J. Anticancer therapy targeting the apoptotic pathway. Lancet Oncol, 2003, 4: 721-729

42 Li Y H, Li X L, Wong Y S, et al. The reversal of cisplatin-induced nephrotoxicity by selenium nanoparticles functionalized with 11-mercapto-1-undecanol by inhibition of ROS-mediated apoptosis. Biomaterials, 2011, 32: 9068-9076

43 Huang $\mathrm{C} \mathrm{H}$, Jin $\mathrm{H}$, Song B, et al. The cytotoxicity and anticancer mechanisms of alterporriol $\mathrm{L}$, a marine bianthraquinone, against MCF-7 human breast cancer cells. Appl Microbiol Biotechnol, 2012, 93: $777-785$

44 Wang X. The expanding role of mitochondria in apoptosis. Genes Dev, 2001, 15: 2922-2933

45 Salganik R I. The benefits and hazards of antioxidants: Controlling apoptosis and other protective mechanisms in cancer patients and the human population. J Am Coll Nutr, 2001, 20: 464S-472S

46 Shackelford R E, Kaufmann W K, Paules R S. Oxidative stress and cell cycle checkpoint function. Free Radic Biol Med, 2000, 28: 1387-1404

47 Ma L N, Song B, Jin H, et al. Cinobufacini induced MDA-MB-231 cell apoptosis-associated cell cycle arrest and cytoskeleton function. Bioorg Med Chem Lett, 2012, 22: 1459-1463

48 Paul S B, Yisang Y, James L R, et al. Calcium, ATP, and ROS: A mitochondrial love-hate triangle. Am J Physiol Cell Physiol, 2004, 287: 817-833

49 Shih C M, Ko W C, Wu J S, et al. Mediating of caspase-independent apoptosis by cadmium through the mitochondria-ROS pathway in MRC-5 fibroblasts. J Cell Biochem, 2004, 91: 384-397

50 Nicotera P, Bellomo G, Orrenius S. Calcium-mediated mechanisms in chemically induced cell death. Annu Rev Phar-Macol Toxicol, 1992, 32: 449-470

51 Melanie H, Amanda M G V, Pieter S, et al. Rho-kinase-dependent $\mathrm{F}$-actin rearrangement in involved in the inhibition of PI3-kinase/Akt during ischemia-reperfusion-induced endothelial cell apoptosis. Apoptosis, 2008, 13: 404-412

52 Olivia N T, Jocelyne H, Jacqueline B. Cytoskeleton and apoptosis. Biochem Pharmacol, 2008, 76: 11-18

Open Access This article is distributed under the terms of the Creative Commons Attribution License which permits any use, distribution, and reproduction in any medium, provided the original author(s) and source are credited. 